\title{
Neutrino Scattering in Perturbative QCD and Implications for the Weinberg Angle
}

\author{
Stefan Kretzer* and Mary Hall Reno ${ }^{\dagger}$ \\ ${ }^{*}$ Physics Department and RIKEN-BNL Research Center, Brookhaven National Laboratory, \\ Upton, New York 11973, U.S.A. \\ ${ }^{\dagger}$ Department of Physics and Astronomy, University of Iowa \\ Iowa City, Iowa 52242 USA
}

\begin{abstract}
We summarize a recent calculation of perturbative neutrino cross sections that includes NLO and mass corrections. We provide numerical results for quantities that are related to the extraction of the weak mixing angle from neutrino deep inelastic scattering.
\end{abstract}

\section{NEUTRINO CROSS SECTIONS IN PERTURBATIVE QCD}

At neutrino energies above a few $\mathrm{GeV}$, neutrino nucleon cross sections are dominated by deep inelastic interactions that are perturbatively assessable in QCD [1]. In Ref. [2] we calculated

- $\mathscr{O}\left(\alpha_{s}\right)$ perturbative next-to-leading order (NLO) corrections

- $\mathscr{O}\left(M^{n} / Q^{n}\right)$ target mass effects

- $\mathscr{O}\left(m^{n} / Q^{n}\right)$ and $\mathscr{O}\left(\ln m^{2} / Q^{2}\right)$ heavy quark mass effects

- $\mathscr{O}\left(m_{l}^{2 n} /\left(M^{n} E_{l}^{n}\right)\right)$ heavy lepton mass effects (mostly for $\tau$ production)

by combining the OPE technique of Georgi \& Politzer [3] with the 1-loop corrections in Refs. [4]. One could summarize the above list as $\xi$-scaling for weak structure functions merged with NLO QCD for light and heavy quarks: While the heavy quark and perturbative corrections come in through the expansion of the Wilson coefficients in the operator product expansion, the target mass corrections enter through the Lorentz structure of the corresponding (non-reduced) operators. In this contribution to the proceedings, we will apply the calculation in Ref. [2] to quantities that are related to the weak mixing angle analysis in Ref. [5] which found a value of $\sin ^{2} \Theta_{\mathrm{W}}$ deviating by $\sim 3 \sigma$ from the standard model expectation.

\section{THE PASCHOS-WOLFENSTEIN RELATION AND $R^{v, \bar{v}}$}

In the following, integrated neutral current $(\mathrm{NC})$ and charged current $(\mathrm{CC})$ cross sections

$$
\sigma_{\mathrm{NC}, \mathrm{CC}}^{v, \bar{\nu}}=\frac{\left.\int d E_{\nu, \bar{v}} d \sigma_{\mathrm{NC}, \mathrm{CC}}^{v, \bar{v}} \Phi\left(E_{\nu \bar{v}}\right)\right|_{20 \mathrm{GeV}<y E_{v, \bar{v}}<180 \mathrm{GeV}}}{\int d E_{\nu, \bar{v}} \Phi\left(E_{\nu \bar{v}}\right)}
$$


will refer to flux-averaged integrals with a cut on hadronic energy as in the experimental analysis [5]. We will consider the counting experiment observables

$$
R^{v, \bar{v}} \equiv \frac{\sigma_{\mathrm{NC}}^{v, \bar{v}}}{\sigma_{\mathrm{CC}}^{v, \bar{v}}}
$$

as well as the Paschos-Wolfenstein [6] relation

$$
R^{-} \equiv \frac{\sigma_{\mathrm{NC}}^{v}-\sigma_{\mathrm{NC}}^{\bar{v}}}{\sigma_{\mathrm{CC}}^{v}-\sigma_{\mathrm{CC}}^{\bar{v}}} \simeq \frac{1}{2}-\sin ^{2} \Theta_{\mathrm{W}}
$$

For an ideally iso-scalar target and under the neglect of charm production components one has that the approximation Eq. (3) is exact at arbitrary order in QCD as long as isospin symmetry is exact and $(s-\bar{s})(x)=0$ holds for the nucleon's (anti-)strange quark parton distribution functions [7]. For definiteness, we will consider the scattering of neutrinos on an $Z=26, A=56$ iron target and charm production with $m_{c}=1.3 \mathrm{GeV}$. We assume $(s-\bar{s})(x)=0$ to focus on the NLO and mass corrections.

Our results for $R^{v, \bar{v}}$ are summarized in Table 1 for two sets of parton distribution functions [8, 9], for a LO or NLO evaluation and for the standard model and the anomalous value of the Weinberg angle. In the first line, the numbers in parentheses refer to a perturbative expansion of the ratios $R^{v, \bar{v}}$ directly (instead of the ratios of perturbatively expanded cross sections in Eq. (2)); i.e. schematically $R^{v}=R_{(0)}^{v}+\alpha_{S} R_{(1)}^{v}$ inside the parentheses. These results can be summarized as follows

- $R^{\bar{v}}$ is insensitive to the Weinberg angle and sensitive to NLO corrections

- $R^{v}$ is insensitive to NLO corrections within its sensitivity to the Weinberg angle

- The impact of PDF uncertainties from pre-determined PDF fits is inconclusive, e.g. the error estimate of CTEQ6 for $R^{v}$ does not overlap with the evaluation based on GRV.

From these results, one cannot derive a conclusive estimate of the impact of NLO corrections on the analysis [5] and further work will be required. For now, we will restrict ourselves to playing the game to treat Eq. (3) as a would-be identity and to solve it for the Weinberg angle:

$$
\begin{aligned}
\frac{1}{2}-\left.R^{-}\right|_{\left\{\text {GRV LO, } \sin ^{2} \Theta_{\mathrm{W}}=0.2227\right\}} & =0.2192(3) \\
\frac{1}{2}-\left.R^{-}\right|_{\left\{\text {GRV NLO, } \sin ^{2} \Theta_{\mathrm{W}}=0.2227\right\}} & =0.2192(2) \\
\frac{1}{2}-\left.R^{-}\right|_{\left\{\text {CTEQ NLO, } \sin ^{2} \Theta_{\mathrm{W}}=0.2227\right\}} & =0.2196(9) \pm 0.0005(1)
\end{aligned}
$$

The difference between the numerical values in Eqs. (4) and Eqs. (5) reflects the impact of a LO or NLO evaluation of the cross sections entering $R^{-}$. The error quoted with the NLO evaluation using CTEQ6M refers to the master formula (3) in Ref. [9] 
TABLE 1. The ratios $R^{v, \tilde{v}}$ as defined in Eq. (2). Details in the text.

\begin{tabular}{l|l|l} 
PDF $\left(\sin ^{2} \Theta_{\mathrm{W}}\right)$ & $R^{\nu}$ & $R^{\bar{\nu}}$ \\
\hline GRV NLO $(0.2227)$ & $0.3120(0.3115)$ & $0.3844(0.3838)$ \\
GRV LO $(0.2227)$ & 0.3125 & 0.3860 \\
GRV NLO (0.2277) & 0.3088 & 0.3839 \\
CTEQ6 NLO (0.2227) & $0.3105 \pm 0.0006$ & $0.3841 \pm 0.0038$
\end{tabular}

and has to be understood as explained in detail in this reference. For the observable $R^{-}$we find a very robust stability under NLO corrections. We also find a similar stability with regards to PDF variations as long as they do not exploit any new physical degree of freedom such as isospin violations or $(s-\bar{s})(x) \neq 0$. Note, however, that $(s-\bar{s})(x) \neq 0$ has to be expected in general because there is no symmetry that would protect $(s-\bar{s})(x)=0$. The consequences of this strange sea asymmetry will be discussed in [10].

\section{ACKNOWLEDGMENTS}

S.K. is grateful to RIKEN, Brookhaven National Laboratory and the U.S. Department of Energy (contract No. DE-AC02-98CH10886) for providing the facilities essential for the completion of this work. The work is also supported by the U.S. Department of Energy under Contract No. FG02-91ER40664.

\section{REFERENCES}

1. E. A. Paschos and J. Y. Yu, Phys. Rev. D 65, 033002 (2002); S. Kretzer and M. H. Reno, Phys. Rev. D 66, 113007 (2002); H. M. Gallagher and M. C. Goodman, NuMI note NuMI-112 (1995), http://www.hep.anl.gov/ndk/hypertext/numi_notes.html; and references in these articels.

2. S. Kretzer and M. H. Reno, hep-ph/0307023.

3. H. Georgi and H. D. Politzer, Phys. Rev. D 14, 1829 (1976); A. De Rújula, H. Georgi, and H. D. Politzer, Ann. Phys. 103, 315 (1977).

4. G. Altarelli, R. K. Ellis and G. Martinelli, Nucl. Phys. B 157, 461 (1979); T. Gottschalk, Phys. Rev. D 23 (1981) 56; W. Furmanski and R. Petronzio, Z. Phys. C 11, 293 (1982); M. Glück, R.M. Godbole and E. Reya, Z. Phys. C38 (1988) 441; 39 (1988) 590 (E). M. Glück, S. Kretzer and E. Reya, Phys. Lett. B 380, 171 (1996); B 405, 391 (1996) (E).

5. NuTeV Collaboration, G.P. Zeller et al., Phys. Rev.Lett. 88, 091802, 2002; and K. McFarland's contribution to these proceedings.

6. E.A. Paschos and L. Wolfenstein, Phys. Rev. D 7, 91 (1973).

7. S. Davidson, S. Forte, P. Gambino, N. Rius, A. Strumia, JHEP 0202, 037, 2002.

8. M. Glück, E. Reya and A. Vogt, Eur. Phys. J. C 5, 461 (1998) [arXiv:hep-ph/9806404].

9. J. Pumplin, D. R. Stump, J. Huston, H. L. Lai, P. Nadolsky and W. K. Tung, JHEP 0207, 012 (2002).

10. S. Kretzer, F. Olness, J. Pumplin, M.H. Reno, D. Stump and W.-K. Tung, article under preparation. 